\title{
Driver Assistance System for Collision Avoidance
}

\author{
T N Farheen \\ PG Scholar \\ Dept. of Electronics and Communication \\ Ballari Institute of Technology and Management \\ Ballari, Karnataka, India
}

\author{
Shiva Kumar K S \\ Assistant Professor \\ Dept. of Electronics and Communication \\ Ballari Institute of Technology and Management \\ Ballari, Karnataka, India
}

\begin{abstract}
Advanced Driver Assistance System (ADAS) is been most innovative and advanced technology in the automobile industry so far from the past decade. Though the technology emerges there also exist a gradual increase in the road accidents major of these are due driver negligence. The paper proposes a novel methodology for avoiding such mishaps and collisions using an advanced technology in a low cost method. Here we implement a method to do three basic functionalities such as, collision avoidance, lane change detection and liquor detection of the driver and give a best safety action of all the situations. We cast off the system with an efficient, low cost Mega 2560 controller along with the required sensors. Collision avoidance is implemented based on adaptive cruise control system. The system is been tested with the maximum test cases in real time prototype model and the results are present in the paper. In future the system can be added with additional situations and give an alert to the driver or the concerned persons.
\end{abstract}

Keywords- ADAS, collision, Cruise control, Mega, microcontroller

\section{INTRODUCTION}

Based on WHO, 1.26 million deaths were logged due to road mishap in 2014. Receiving a newsflash of road mishap is very communal these days. Road mishaps are very significant, and from overhead cited statistics, we can comprehend that several guiltless persons expire in road mishap every year. Though, there remain numerous unreceptive accident security schemes similar airbag, seat belts. Assistance in saving life afterwards crash otherwise mishap have happened. Therefore, there exist great request of an idea that be able to stop the vehicles from mishap. ADAS is a system that is right essential of nowadays road. ADAS is a scheme that offers motorists a sympathetic of support during driving. It assistances drivers to notice lane, to escape crash with walkers, in addition to it likewise assistances the driver to identify the street traffic signs, henceforth traffic guidelines can be charted correctly.

Maximum road mishaps happen due to humanoid mistake. ADAS are schemes industrialized to systematize, adapt, and improve vehicle schemes for protection and improved driving. ADAS automatic schemes are confirmed to decrease road mortalities by diminishing humanoid mistake. Security features are intended to evade calamities and crashes by proposing tools that aware the driver to glitches, applying protections, and enchanting governor of automobile if essential. Adaptive structures may computerize lighting, deliver adaptive cruise regulator, contribution in escaping crashes, integrate traffic cautions, attentive motorists to potential difficulties, assist in track leaving and lane positioning, provide directional support through smart phones, in addition deliver many additional features.

\section{EXISTING METODS}

Few prevailing methods to avoid road mishaps embrace antilock braking system, Supplementary restraint scheme airbags, Electronic brake-force dissemination, Immobilizer, Cruise control, parking sensors. We brief out few recent proposed methodologies in according to the ADAS

\section{A. A FCW scheme by CNN [1]}

Shivam Kumar et.al proposed an FCW method is to contemporary a real-world explanation for all street car mishaps taking place. The prototypical monitors the forgoing cars and concurrently it will compute distance amongst the automobiles. Now, if forgoing automobile gets very close, then the prototypical will caution that driver for imminent bang. Driver of automobile will just be warned by some noticeable signal afterward getting onset distance amongst automobiles. The prototypical will remain precise ample useful, particularly for beginners. This scheming of distance amongst automobiles will be completed by CNN founded prototypical and camera that situated on wind shield to clack back side imageries of forgoing automobile in real time. In their investigational outcomes, they found that the prototypical can compute distance and can be capable to attain a complete worthy accurateness. In preceding, scholars castoff these skills like FCW and ADAS arrangement which are completely reliant on the hardware besides lucrative as well. Thus, they use CNN in FCW scheme in its place of using the hardware similar to radars.

\section{B. Intelligent Transportation System [2]}

Ashwini Gade, et.al, proposes a low price, moveable and elastic vehicle safety scheme. It imparts the usage of an embedded micro-web server in Raspberry-pi microcontroller, through IP connectivity for distantly controlling the maneuvers from alternative position. The projected scheme does not necessitate a steadfast server PC w.r.t. alike methods and offers an offbeat station to best and associate the vehicle atmosphere with more than fair the swapping functionality. The scheme for its viability and efficiency will be combined with external strategies such as gas, alcohol, ultrasonic and pressure sensors. All overhead topographies will forecast the scheme to system an intelligent transport scheme for a cleverer and additional protected way of travelling.

\section{ADAS [3]}

Gopalakrishnan, et.al, proposes method to stop over fastmoving, automobile crashes, and driver attentiveness schemes are discussed. RFID readers are castoff for detecting the speed boundary in signposts. The speediness of automobile is accomplished founded on interpretation gotten from tags. 
Devices like alcohol, ultrasonic, gas, temperature sensor are castoff to measure other limitations to improve the security measure while driving.

\section{Real-Time FCA using Pi [4]}

Wai Chun Phoon et.al, proposed an instantaneous low cost Frontward Crash Attentive Scheme (FCAS). FCAS are talented to vigilant drivers, once their carriages are attainment too near to anterior automobile by approximating the rapidity of automobile in anterior, by means of a lurid bleeping sound. The FCAS contains of five chief stages, such as, audiovisual attainment, Speed gaining, ROI segmentation, Automobile discovery, and RPD Attentive Scheme. The FCAS is entrenched on a Raspberry-Pi for real-time. Investigational outcomes demonstrations that organization can dependably attentive the motorist, in real-time, once a touching automobile in obverse location is besides adjacent.

\section{E. Fuzzy Logic founded Collision Avoidance Scheme [5]}

Hossam Elsayed et.al proposed a fuzzy lucidity controller method is for crash avoidance security (CAS) scheme with self-driving automobile steering regulator. The steering supervisor stretches the anticipated tires angle to retain our automobile on accurate track till attainment goal opinion while handling the 2D laser data in circumstance of hurdle or pedestrian recognition prominent to safety directional or abruptly stop. Investigational exertion was achieved on GAZEBO simulation tool and supervisor was intended and employed on MATLAB Simulink. The outcomes show the controller delivers an important development in decision creation while navigation.

\section{PROPOSED METHODOLOGY}

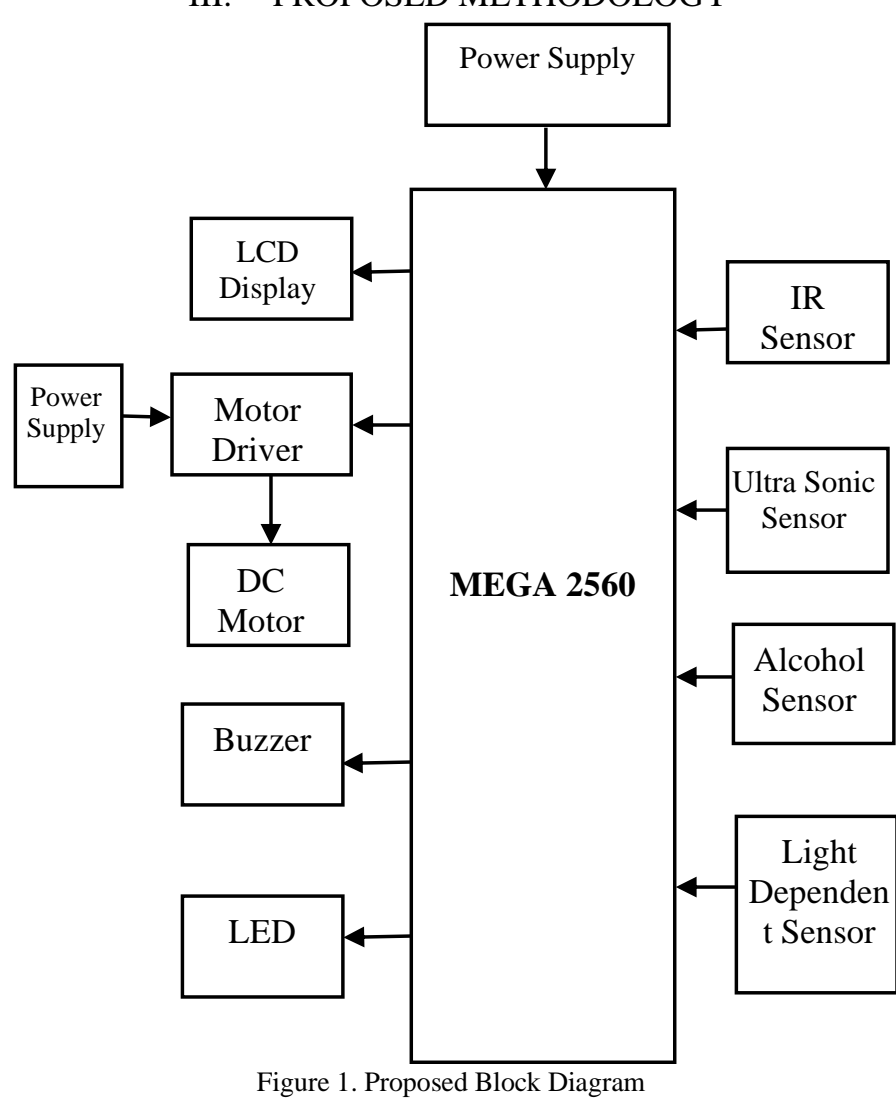

The various components and sensors required for the complete implementation of the modules are connected to the microcontroller board which is be displayed in the figure 1 . The list of components and sensor included in the module are Mega 2560 controller, Light Dependent (LDR), Ultra sonic Sensor, Light Emitting Diode (LED), Buzzer, Liquid Crystal Display (LCD), motor control setup and power supply. We elaborate the working of each module and sensor in detail as follows.

\section{A. Microcontroller-Arduino MEGA 2560}

Mega 2560 is a microcontroller panel founded on ATmega2560. It partakes 16 analog inputs, 54 alphanumeric IO pins, 4 UART, one USB assembly, $16 \mathrm{MHz}$ crystal oscillator, an ICSP header, one power jack and a reset button. It comprises the whole thing required to provision the microcontroller; merely connect to a mainframe with USB cable or control it through an AC2DC connecter or battery to get happening. This panel is well-matched with maximum shields intended for Uno and previous panels Diecimila.

\section{B. Ultra Sonic Sensor}

In common, Ultrasonic devices are founded on the measurement of possessions of acoustic surfs with frequencies beyond the humanoid audible variety, regularly at bumpily 41 $\mathrm{kHz}$. They characteristically function by producing a highfrequency pulse of sound, getting and appraising the possessions of reverberation pulse. Ultrasonic device unit SRF04 delivers 3 to $410 \mathrm{~cm}$ non-contact dimension gathering, the vacillating accurateness can spread to $2.5 \mathrm{~mm}$. The component comprises ultrasonic sources, receiver and controller circuit in a solitary package.

\section{Alcohol Sensor - MQ3 gas sensor}

The MQ3 gas device is a liquor sensor that is castoff to identify the alcohol concentration on our breath. The device delivers an analog resistive outcome founded on alcohol attentiveness. When the liquor gas occur, the sensor's conductivity becomes greater along with gas attentiveness increasing. It is appropriate for numerous submissions of noticing alcohol at dissimilar concentration.It is extensively castoff in domestic liquor gas alarm, engineering alcohol gas panic and moveable liquor detector.

\section{D. $L D R$}

The LDR is a submissive constituent that diminutions resistance regarding getting luminosity on module's sensitive superficial. LDR Device Component is castoff to detect the incidence of light by calculating the strength of light. The outcome of the unit goes high in incidence of light and it develops low in the nonexistence of light. The feeling of the gesture discovery can be accustomed using potentiometer.

The adaptive cruise control system is implemented for collision avoidance system. The ultrasonic sensor is detects the distance between preceding vehicle and the installed vehicle. Based on the distance calculated a decision is taken either to proceed further or slow down or if necessary to stop. Buzzer is been turned on if the distance is very low and vehicle is slowed down. The flow of the complete process is displayed in figure 2 below. 


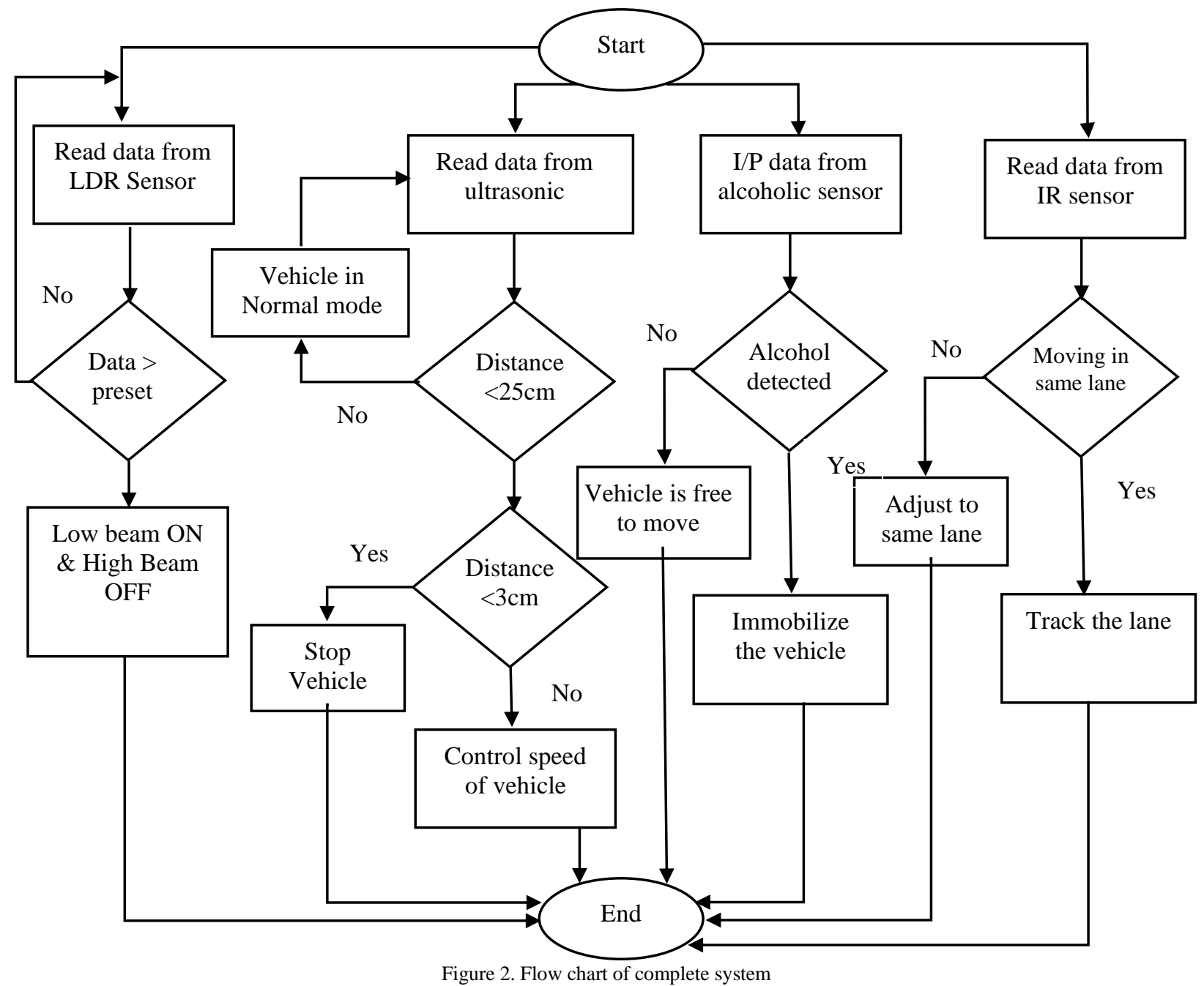

Based on the MQ3 gas sensor the vehicle is automatically turned ON or OFF. If there is any presence of alcohol content in the driver the vehicle is automatically turn OFF.

The LDR sensor gives the level of light intensity present outside the vehicle i.e. detects whether any opposite vehicle exists or not. If there is any vehicle the lights of the running vehicle is automatically made low beam. This basically prevents accidents due to glaring effect.

The last module detects the lane change over and lane tracking based on the IR sensor output the tracking and lane change over is been detected.

\section{RESULTS AND DISCUSSION}

Here we display most of the results of our module. The figure 3 display the completed picture of the implemented module with all the components integrated. The Figure 4 displays the normal mode of working of the module where the distance between the two vehicles is inside defined threshold, once the distance reduces below the threshold the vehicle automatically turns to cruise mode which is displayed in figure 5. In the stage if the distance is still more less the vehicle is automatically turned off as displayed in figure 6 . The glaring effect working is displayed in figure 7 and 8 by turning on low and high beams when required. The figure 9 displays the detection of alcohol. The lane departure warning system is detected based on IR sensor incorporated as shown in figure 10.

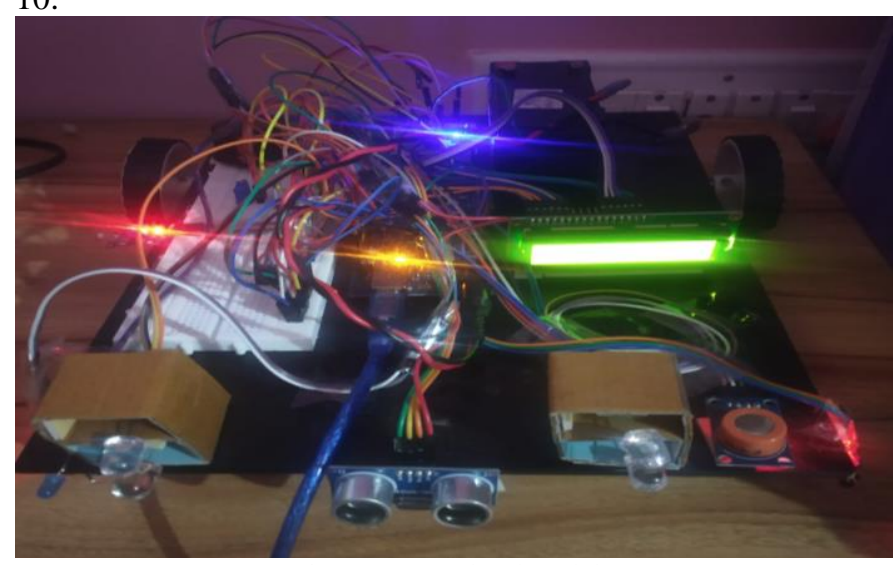

Figure 3. Completed module

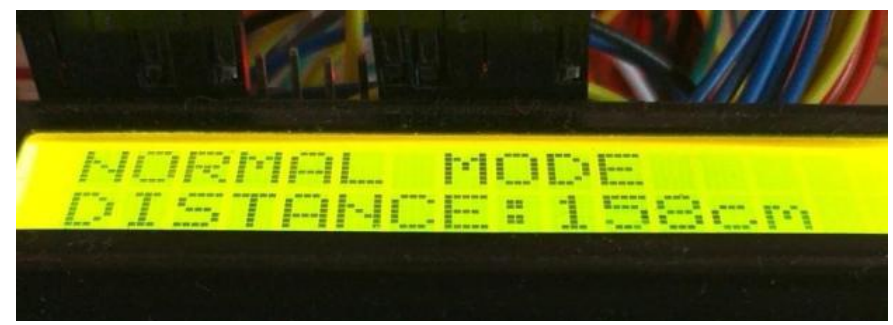

Figure 4. Normal Mode 


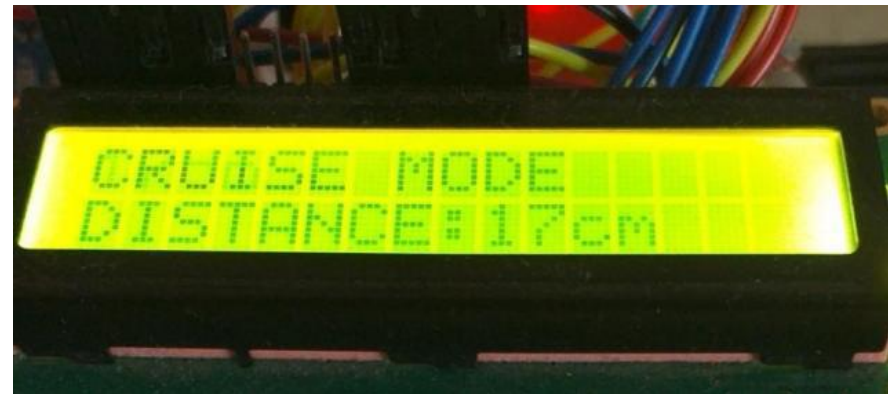

Figure 5. Cruise Mode

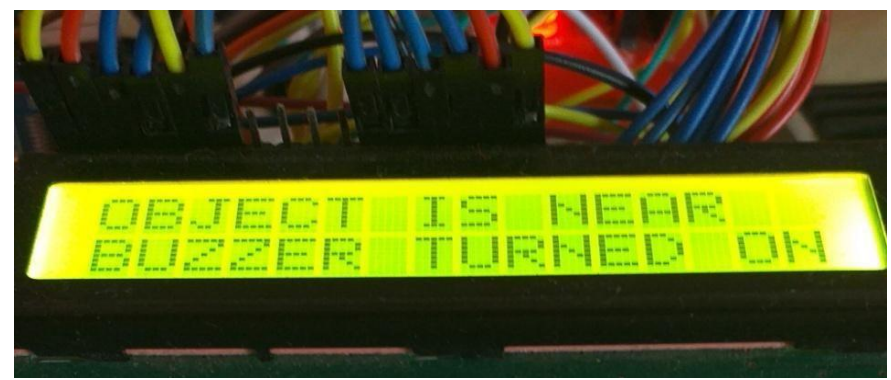

Figure 6. Near to an object or Vehicle

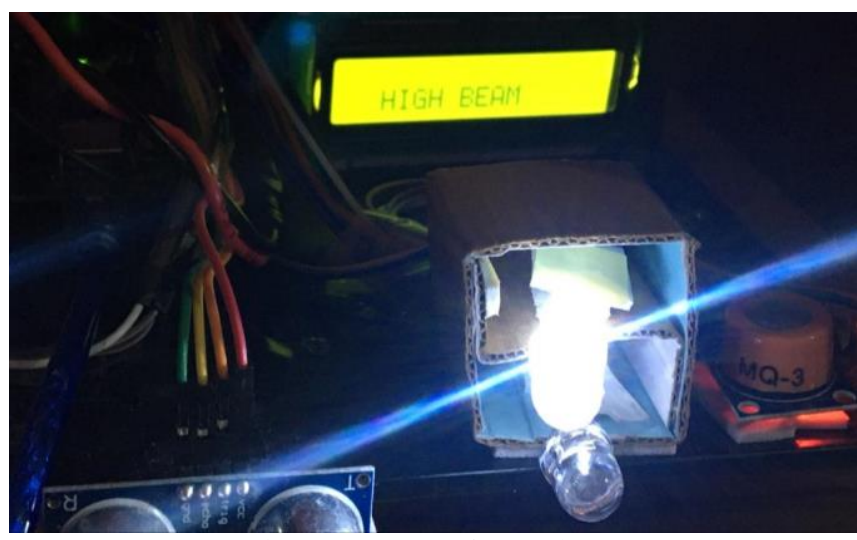

Figure 7. Turn on of HIGH beam

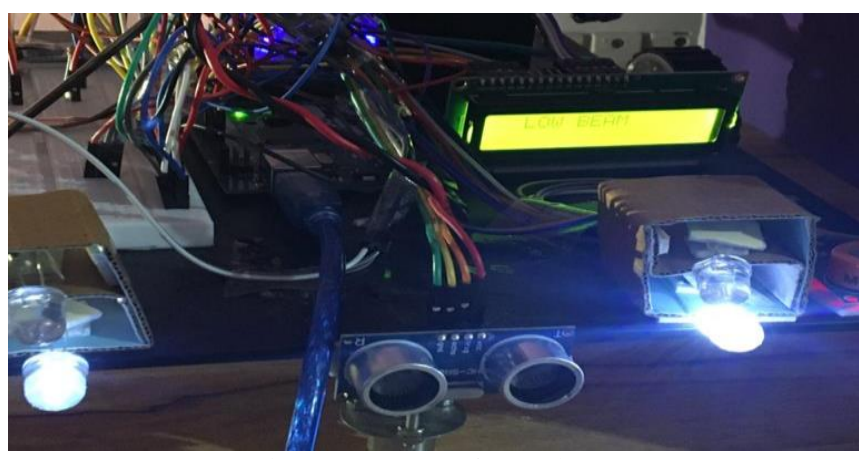

Figure 8. Turn on of LOW beam

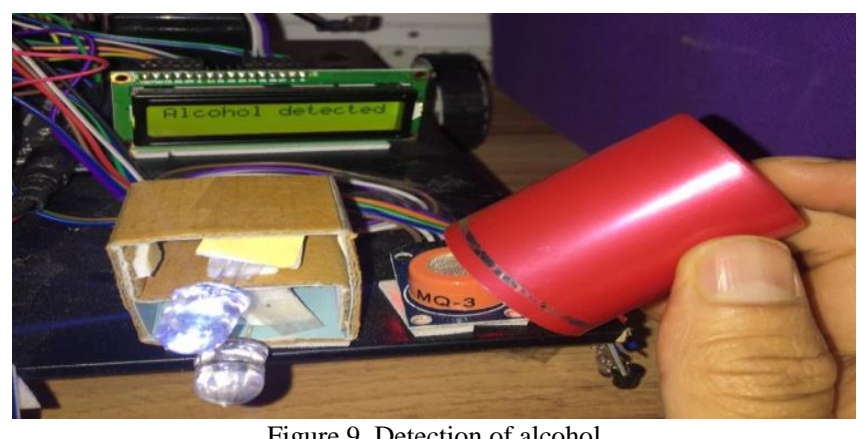

Figure 9. Detection of alcohol

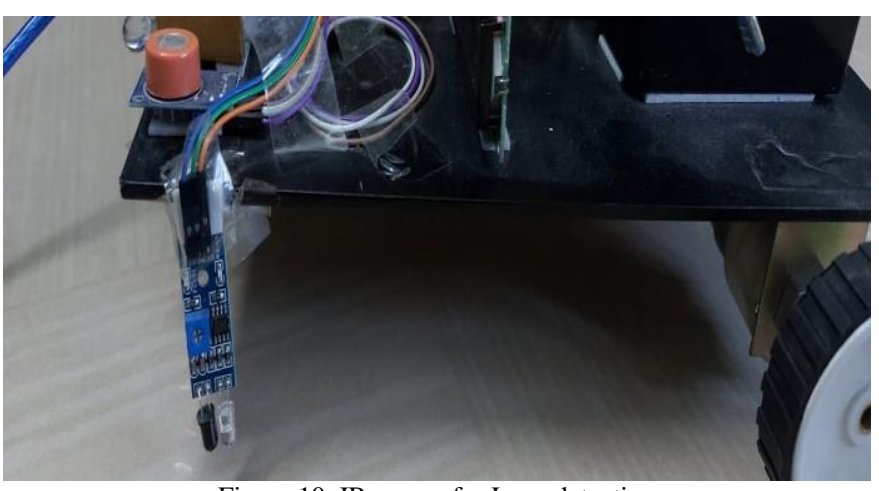

Figure 10. IR sensor for Lane detection

\section{CONCLUSION}

The adaptive cruise control system being a crucial part of the complete system, as a prototype point of view the vehicle was speed was decreased or stopped. This in real time implementation may lead to some other mishaps or accidents when the vehicle stops, vehicle behind may hit. The model was working successful as per the expected outcomes. Each and every module was tested separately and later integrated to the system. The alcohol detection system can decrease major of the accidents in real time.

\section{REFERENCES}

[1] S. Kumar, V. Shaw, J. Maitra and R. Karmakar, "FCW: A Forward Collision Warning System Using Convolutional Neural Network," 2020 International Conference on Electrical and Electronics Engineering (ICE3), Gorakhpur, India, 2020, pp. 1-5, doi: 10.1109/ICE348803.2020.9122858.

[2] Ashwini Gade, Rasika Khollam, Karenca Fichardo, "Intelligent Transportation System", International Journal of Recent Technology and Engineering (IJRTE), ISSN: 2277-3878, Volume-9, Issue-2, July 2020, pp.590-593, DOI:10.35940/ijrte.B3578.079220

[3] K. Gopalakrishnan, S. Afrin Fathima, K. S. Matheshwari, "Advanced Driver Assistance System", International Journal of Recent Technology and Engineering (IJRTE), ISSN: 2277-3878, Volume-8 Issue-6, March 2020, pp. 3481-3487, DOI:10.35940/ijrte.F8900.038620.

[4] W. C. Phoon and P. Y. Lau, "Real-Time Forward Collision Alert System using Raspberry Pi," 2019 International Symposium on Intelligent Signal Processing and Communication Systems (ISPACS), Taipei, Taiwan, 2019, pp. 1-2, doi: 10.1109/ISPACS48206.2019.8986282.

[5] H. Elsayed, B. A. Abdullah and G. Aly, "Fuzzy Logic Based Collision Avoidance System for Autonomous Navigation Vehicle," 2018 13th International Conference on Computer Engineering and Systems (ICCES), Cairo, Egypt, 2018, pp. 469-474, doi: 10.1109/ICCES.2018.8639396.

[6] S. Sarala, D. S. Yadav, and A. Ansari, "Emotionally Adaptive Driver Voice Alert System for Advanced Driver Assistance System (ADAS) Applications," in Proceedings of the 2018 International Conference on Smart Systems and Inventive Technology (ICSSIT). IEEE, 2018, pp. 509-512.

[7] S. M. Iranmanesh, H. N. Mahjoub, H. Kazemi, and Y. P. Fallah, "An Adaptive Forward Collision Warning Framework Design Based on river Distraction," IEEE Transactions on Intelligent Transportation Systems, vol. 19, no. 12, pp. 3925-3934, 2018.

[8] Paolo Bosetti, Mauro Da Lio, Andrea, Saroldi, On Curve Negotiation from Driver Support to Automation, IEEE Transaction on Intelligent Transportation Systems, 2015, Volume 16, Issue 4.

[9] 4. Chaudhari Priyanka Ramnath, Advanced Driver Assistance System, International Journal of Advanced Research in Electronics and Communication Engineering, 2015, Volume 4, Issue 10.

[10] Arun Tigadi, Rudrappa Gujanatti, Anil Gonchi, Advanced Driver Assistance System, International Journal of Engineering Research and General Science, May-June 2016, Volume 4, Issue 3, 2019-2730. 